\title{
Exclusive Breastfeeding; How Could It Be Promoted?
}

\author{
Najmeh Maharlouei (10 ${ }^{1, *}$ \\ ${ }^{1}$ Health Policy Research Center, Institute of Health, Shiraz University of Medical Sciences, Shiraz, Iran \\ "Corresponding author: Health Policy Research Center, Institute of Health, Shiraz University of Medical Sciences, Shiraz, Iran. Email: najmeh.maharlouei@gmail.com
}

Received 2019 June 22; Accepted 2019 June 28.

Keywords: Breastfeeding, Lactation, Increase

Nutritional status from conception to the end of the second year of life plays a critical role in the health of the entire life (1). The importance of this golden period of 1000 days is very remarkable and it can affect the new generations' wellbeing (2). This "window of opportunity" is more important in low and middle-income countries where not only food quantity but also its quality is not satisfactory. Simultaneously, babies in this period need macro- and micro-nutrients much more than adults do (1).

Breast milk is considered the most appropriate source of nutrition for newborns. According to the World Health Organization, exclusive breastfeeding, vaccination, and some vitamins are the only vital elements determining the newborns' health (3). Numerous studies have proven the benefits of breastfeeding during childhood $(4,5)$ and adulthood (6). Moreover, lactating mothers would experience better physical and mental health $(7,8)$.

Surveys show that the prevalence of exclusive breastfeeding during the first six months of life varies widely among countries. For example, in the urban setting of Ethiopia, the prevalence of exclusive breastfeeding was reported as $60.9 \%$ (95\% CI: 56.6\% - 65.1\%) (9). However, a systematic review concluded that in Middle East countries, only $20.5 \%$ (95\% CI: $14.5 \%-28.2 \%$ ) of babies were exclusively breastfed for the first six months of life (10). On the other hand, the results of a population-based cohort study (11) on lactating mothers in Iran showed that the mean duration of exclusive breastfeeding was $4.63 \pm 1.99$ months (12).

Different factors can affect the practice of exclusive breastfeeding during the first six months of life. Studies have demonstrated that a supportive environment, especially the positive attitude of the partner regarding breastfeeding, would remarkably accentuate the duration of exclusive breastfeeding (13). Moreover, mothers who receive counseling regarding the benefits of exclusive breastfeeding and its techniques are more likely to maintain exclu- sive breastfeeding for six months (14). In addition, various maternal factors would remarkably affect the duration of exclusive breastfeeding. Of the most frequently reported factors are maternal post-partum infections, breast complications, cesarean section by maternal request, mothers' full-time employment, birthplace (hospitals versus facility centers), maternal perception of insufficiency of breast milk, and twin pregnancies $(9,12,14,15)$. Besides, babies who are irritable and those who have less satisfactory weight gain during infancy are less benevolent from breastfeeding. On the other hand, those babies who suck pacifiers are more likely to be fed on formula $(9,10,12-$ 14). There are two proposed hypotheses behind this fact. The first one would be nipple confusion as the shapes of most pacifiers differ from nipple anatomy. The other reason would be the fact that by using pacifiers, babies satisfy their sucking desire, which may result in less stimulation of the nipple by the babies. Consequently, less prolactin hormone secretion from the posterior pituitary gland would lead to the inadequacy of breast milk quantity.

In conclusion, promoting exclusive breastfeeding should be considered as a multi-disciplinary program that needs the collaboration of different sectors.

Lactating mothers should be benefited from parental leave; consequently, they would devote themselves to provide a tranquilizing atmosphere for their family. For a minority of mothers with critical positions, there should be high-quality nurseries in the workplace so that mothers could feed their infants on demand. Moreover, lactating mothers should be benefitted from some incentives including free gyms and free counseling sessions.

Simultaneously, an advocating multi-dimensional program should be propagated. Fathers should be informed of their principal role in supporting their wives with a positive attitude regarding breastfeeding. Some health volun- 
teers should be trained to support mothers with scientific and precise information regarding breastfeeding. Moreover, mothers should be informed of indicators of breast milk adequacy, as well as definite and simultaneously limited indications of starting formula. In addition, healthcare workers should be motivated to hold meetings advocating the advantages of breastfeeding for not only expecting mothers but also other audiences.

Moreover, there should be some special venues for lactating mothers in public places including the parks, the cinemas, sidewalks, and bus stations. Therefore, it would help mothers to overcome the shy feeling regarding breast feeding in public places.

Therefore, promoting breastfeeding is done by providing a motivational environment where breastfeeding is considered as dignity and value.

\section{Footnotes}

Conflict of Interests: Nothing to be declared.

Funding/Support: This letter was not financially funded by any institution.

\section{References}

1. Adu-Afarwuah S, Lartey A, Dewey KG. Meeting nutritional needs in the first 1000 days: A place for small-quantity lipid-based nutrient supplements. Ann N Y Acad Sci. 2017;1392(1):18-29. doi: 10.1111/nyas.13328. [PubMed: 28319655].

2. Yajnik CS. Transmission of obesity-adiposity and related disorders from the mother to the baby. Ann Nutr Metab. 2014;64 Suppl 1:8-17. doi: 10.1159/000362608. [PubMed: 25059801].

3. World Health Organization. Infant and young child feeding: Model chapter for textbooks for medical students and allied health professionals. Geneva: World Health Organization; 2009.

4. Smith ER, Hurt L, Chowdhury R, Sinha B, Fawzi W, Edmond KM, et al. Delayed breastfeeding initiation and infant survival: A systematic review and meta-analysis. PLoS One. 2017;12(7). e0180722. doi: 10.1371/journal.pone.0180722. [PubMed: 28746353]. [PubMed Central: PMC5528898].

5. Bell S, Yew SSY, Devenish G, Ha D, Do L, Scott J. Duration of breastfeeding, but not timing of solid food, reduces the risk of overweight and obesity in children aged 24 to 36 months: Findings from an Australian cohort study. Int J Environ Res Public Health. 2018;15(4). doi: 10.3390/ijerph15040599. [PubMed: 29587447]. [PubMed Central: PMC5923641].

6. Flohr C, Henderson AJ, Kramer MS, Patel R, Thompson J, Rifas-Shiman SL, et al. Effect of an intervention to promote breastfeeding on asthma, lung function, and atopic eczema at age 16 years: Follow-up of the PROBIT randomized trial.JAMA Pediatr.2018;172(1). e174064. doi: 10.1001/jamapediatrics.2017.4064. [PubMed: 29131887]. [PubMed Central: PMC6583261].

7. Unar-Munguia M, Torres-Mejia G, Colchero MA, Gonzalez de Cosio T. Breastfeeding mode and risk of breast cancer: A dose-response metaanalysis. J Hum Lact. 2017;33(2):422-34. doi: 10.1177/0890334416683676. [PubMed: 28196329].

8. Sipsma HL, Ruiz E, Jones K, Magriples U, Kershaw T. Effect of breastfeeding on postpartum depressive symptoms among adolescent and young adult mothers. J Matern Fetal Neonatal Med. 2018;31(11):1442-7. doi: 10.1080/14767058.2017.1319351. [PubMed: 28412876].

9. Adugna B, Tadele H, Reta F, Berhan Y. Determinants of exclusive breastfeeding in infants less than six months of age in Hawassa, an urban setting, Ethiopia. Int Breastfeed J. 2017;12:45. doi: 10.1186/s13006017-0137-6. [PubMed: 29142586]. [PubMed Central: PMC5669024].

10. Alzaheb RA. A review of the factors associated with the timely initiation of breastfeeding and exclusive breastfeeding in the Middle East. Clin Med Insights Pediatr. 2017;11:1.1795565177489E+15. doi: 10.1177/1179556517748912. [PubMed: 29317851]. [PubMed Central: PMC5753894].

11. Maharlouei N, Atefi S, Lankarani K. A study protocol to follow a birth cohort: Fars birth cohort. Shiraz E Med J. 2018;20(2). e84308. doi: 10.5812/semj.84308.

12. Maharlouei N, Pourhaghighi A, Raeisi Shahraki H, Zohoori D, Lankarani KB. Factors affecting exclusive breastfeeding, using adaptive LASSO regression. Int J Community Based Nurs Midwifery. 2018;6(3):260-71. [PubMed: 30035142]. [PubMed Central: PMC6048001]

13. Ogbo FA, Eastwood J, Page A, Arora A, McKenzie A, Jalaludin B, et al. Prevalence and determinants of cessation of exclusive breastfeeding in the early postnatal period in Sydney, Australia. Int Breastfeed J. 2016;12:16. doi: 10.1186/s13006-017-0110-4. [PubMed: 28405212] [PubMed Central: PMC5385049].

14. Hunegnaw MT, Gezie LD, Teferra AS. Exclusive breastfeeding and associated factors among mothers in Gozamin district, northwest Ethiopia: A community based cross-sectional study. Int Breastfeed J. 2017;12:30. doi: 10.1186/s13006-017-0121-1. [PubMed: 28702071]. [PubMed Central: PMC5504831].

15. Maharlouei N, Mansouri P, Zahmatkeshan M, Lankarani K. Low-risk planned caesarean versus planned vaginal delivery at term: Early and late infantile outcomes. Eastern Mediterranean Health J. 2018. doi: 10.26719/emhj.18.066. 